\title{
Force Spectroscopy of Polyclonal and Monoclonal Anti-Bovine Serum Albumin Antibodies - BSA Complexes
}

\author{
L.A. Chtcheglova* and G. Dietler \\ Institut de Physique de la Matière Condensée, University of Lausanne \\ 1015 Lausanne, Switzerland
}

\begin{abstract}
The specific interactions between bovine serum albumin and poly- or two monoclonal bovine serum albumin antibodies were studied using force spectroscopy mode of atomic force microscopy. The histograms of the unbinding forces for polyclonal bovine serum albumin antibodies are broad at high antibody concentrations ( 50 or $270 \mu \mathrm{g} / \mathrm{ml}$ ) and narrow at low concentrations (10 or $27 \mu \mathrm{g} / \mathrm{ml}$ ), while the histograms for monoclonal antibodies peak at well defined unbinding force. The peak unbinding force depends on the type of antibody and the antibody concentration. In this paper we described and characterized the passive adsorption and covalent immobilization of proteins for tip and sample preparation. Force spectroscopy could serve as a useful method for characterization of antigen-antibody interactions for measuring the specificity of an antibody or to assess the purity of a monoclonal antibody solution and to distinguish between different antibodies.
\end{abstract}

PACS numbers: 82.37.Gk, 82.37.Rs

\section{Introduction}

A basic working principle of molecular recognition processes is based on the specific binding of a ligand molecule (e.g. antibody) to a receptor protein (e.g. antigen). The natural way to know how the molecules interact with each other is a measuring of the rupture force (or unbinding force). In order to investigate antigen-antibody $(\mathrm{Ag}-\mathrm{Ab})$ interactions the atomic force microscope ( $\mathrm{AFM}$ ) operated in force spectroscopy (FS) mode was often used [1, 2].

In this paper, we studied the interaction between polyclonal or two different monoclonal bovine serum albumin (BSA) antibodies with the BSA protein

*corresponding author; e-mail: lilia.chtcheglova@ipmc.unil.ch 
molecule. In our experiments, the antibodies were bound to the tip and the BSA molecules were bound onto the substrate surface.

\section{Materials and methods}

\subsection{Materials}

EDAC (1-ethyl-3 3-dimethylaminopropyl carbodiimide), MES (2-[N-Morpholino] ethanesulfonic acid), APTES (3-aminopropyltriethoxy-silane), BSA and PBS buffer (50 mM phosphate, $150 \mathrm{mM} \mathrm{NaCl}, \mathrm{pH} 7.4$ at $25^{\circ} \mathrm{C}$ ) were purchased from Sigma. The glutaraldehyde was bought from Fluka. The following commercial antibodies were used: the rabbit anti-bovine serum albumin (Sigma, B1520); the monoclonal anti-bovine serum albumin clone BSA-33 (Sigma, B2901) and the monoclonal anti-bovine serum albumin clone BSA1 (ANAWA, 0220-1286), called Ab-BSA, mAb-BSA-33 and mAb-BSA-1, respectively. All reagents were used without further purification. APTES was stored under water-free controlled atmosphere. Aliquots of BSA and all antibodies were prepared and then stored in the freezer at $-20^{\circ} \mathrm{C}$ until use.

Mica was used as substrate for protein attachment. Mica is layered aluminosilicate mineral which is easily cleaved to generate atomically flat surface. The surface of mica has a net negative charge. Many proteins bind tightly to a clean mica surface because of the large surface charge and hydrogen binding between the protein and the surface.

\section{2. $A F M$}

Nanoscope IIIa, Multimode from Digital Instruments (DI) and V-shaped $\mathrm{Si}_{3} \mathrm{~N}_{4}$ (DI) with a nominal spring constant of $0.06 \mathrm{~N} / \mathrm{m}$ were used. Calibration of the cantilevers was performed by the resonance frequency method [3] and thermal fluctuations method [4]. All measurements (recording of force-distance curves and imaging) were carried out in a liquid cell in PBS buffer at ambient temperature. The loading rate was kept at about $3 \mathrm{nN} / \mathrm{s}$ for all force-distance approaches.

\subsection{Tip and sample modification}

Initially, silicon nitride tips and freshly cleaved mica sheets were functionalized with proteins by the simplest method, namely passive adsorption. After submerging into the solution of BSA or antibodies of BSA for several hours at $4^{\circ} \mathrm{C}$, loosely bound proteins were removed by extensive washing. Despite successful and simple use of this method in our experiments, rapid tip degradation and very low event frequency (essentially less than 1\% of all approaches) for antigen-antibody interaction were observed. To increase event frequency without losing biological activity of proteins during experiment a covalent attachment of proteins was carried out. The antibodies were covalently immobilized on the tip using EDAC as 
a coupling agent. Glycine was added in order to neutralize unreacted EDAC. The tips were then washed with PBS buffer. The antibody functionalized tips were used immediately for the measurements.

The protein immobilization onto a substrate surface was preceded by the silanization procedure. Silanes covalently bind to a mica, glass, or Si surface which have silanol $\left(-\mathrm{Si}^{-} \mathrm{O}^{-}\right)$groups present. It was shown that the silanization of mica surfaces by chemical-vapor deposition of alkoxy silanes generally produces a smoother and more stable silane monolayer film (without "vertical" polymerization) than was obtained by liquid-phase deposition $[5,6]$. In our study we followed chemical-vapor deposition of APTES in a vacuum chamber. This procedure was described by Lyubchenko et al. [7, 8]. The activation of mica in vapors of APTES for $2 \mathrm{~h}$ was sufficient to obtain uniformly monolayer. APTES molecules were in an amine-up configuration and about 50\% of these amines were active (protonated, $-\mathrm{NH}_{3}^{+}$) [9]. These reactive amino groups of APTES were covalently coupled with protein amino groups via glutaraldehyde. The silanized substrates were reacted with $1 \%$ ( vol/vol) glutaraldehyde solution in water for 20 minutes and then rinsed several times with water to remove any unreacted glutaraldehyde molecules. Consecutively, the BSA solution (1 $\mathrm{mg} / \mathrm{ml}$ in PBS) was deposited onto the sample surface for 20 minutes at room temperature. The unreacted BSA molecules were removed by washing with PBS buffer.

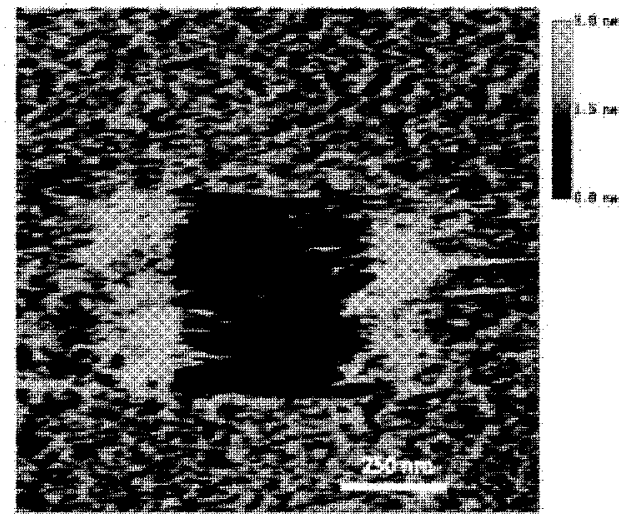

Fig. 1. AFM contact image of the APTES+glutaraldehyde-mica surface. In the central surface area of $500 \times 500 \mathrm{~nm}^{2}$ has undergone scanning at a high loading force (see text).

To check whether APTES was firmly adsorbed on the mica surface and to know the thickness of the silane layer, the sweeping method was used. In this method, the AFM tip first scanned a small part of the surface area (typically, $500 \times 500 \mathrm{~nm}^{2}$ ) with the increased loading force at a scanning speed of $2 \mathrm{~Hz}$. When no further change in the $z$ height of the image was observed, the AFM image was zoomed out a large area (typically, $1.2 \times 1.2 \mu \mathrm{m}^{2}$ ) and rescanned at normal loading forces and scanning speed of $1.5 \mathrm{~Hz}$ (Fig. 1). The APTES+glutaraldehyde layer 
thickness was obtained by measuring the height difference between the altered and the unaltered area in the image. Thus, the layer thickness was roughly estimated and gave a result of about $2 \mathrm{~nm}$. This result was in a good agreement with other experimental data already obtained [9-11].

\subsection{Data analysis}

During FS measurements the cantilever moves down (approach to the surface) and up (retract) and the deflection of the tip is recorded. Knowing the spring constant of the cantilever and variation of the deflection signal per nanometer, one can convert the deflection signal to force for a given deflection. When the tip enters in contact with sample surface, a specific $\mathrm{Ag}-\mathrm{Ab}$ bond can form. This bond will be ruptured when the tip is pulled away from the substrate surface. Therefore, the specific interaction (unbinding) force can be read directly from the force-distance curves as the difference between the equilibrium position and the bottom of the negative peak. To obtain histograms of antigen-antibody forces from force versus distance plots, we used the software developed by Kasas et al. [12].

\section{Results and discussion}

The typical detected force-distance curves (retract part) are shown in Fig. 2. We classify the curves as follows: single bond rupture, non-specific adhesion of tip to sample and no events detected. Most of the curves did not display detectable events, some times display a non-specific adhesion of the tip to surface. The specificity of $\mathrm{Ag}-\mathrm{Ab}$ interaction was verified by blocking the antibody with free antigen. An average extension for all antigen-antibody pairs was determined as $z=14 \pm 3 \mathrm{~nm}$. This value remained the same for our tips and sample functionalization methods. Taking into account the sizes of BSA of $6 \mathrm{~nm}$, antibody

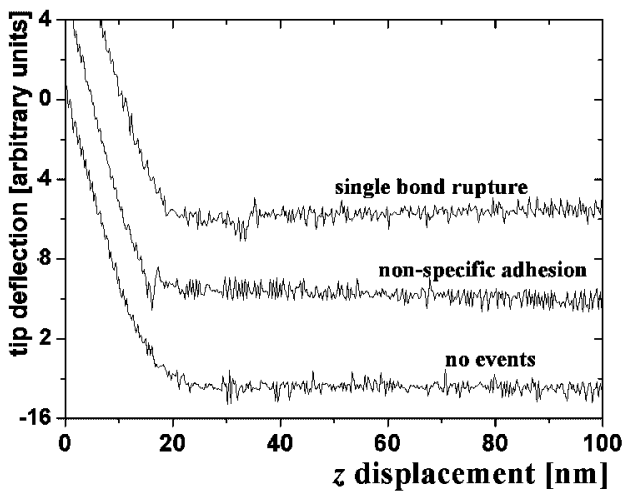

Fig. 2. The typical force-distance curves as measured by AFM. 

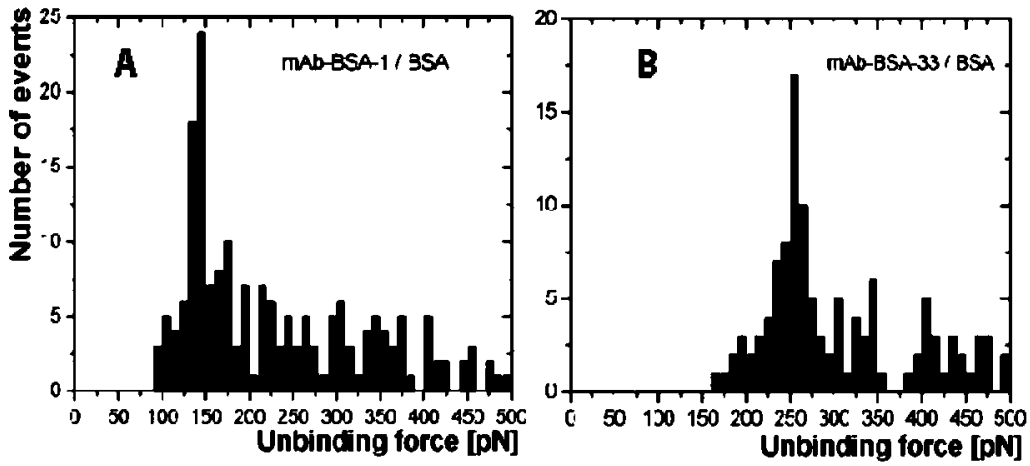

Fig. 3. Histograms of unbinding forces for mAb-BSA-1 - BSA (A) and mA-BSA-33 - BSA (B). Each histogram comprises 640 force-distance curves for the same sample surface area scanned $\left(500 \times 500 \mathrm{~nm}^{2}\right)$. The bin size in the histograms is $10 \mathrm{pN}$.
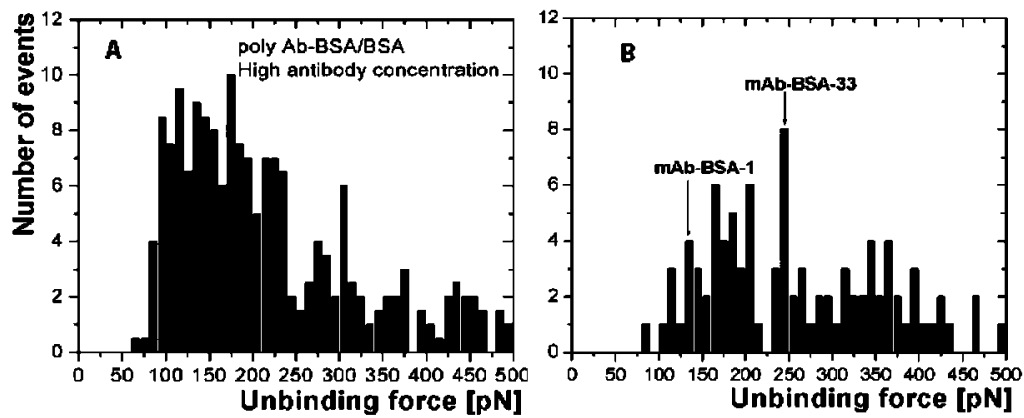

Fig. 4. (A) Histograms of unbinding forces for Ab-BSA - BSA at high antibody concentrations. (B) Histogram of the unbinding forces for the mixture of two monoclonal antibodies - BSA. The total antibody concentration in mixture was kept at high concentration $(270 \mu \mathrm{g} / \mathrm{ml})$. Each histogram comprises 640 force-distance curves for the same sample surface area scanned $\left(500 \times 500 \mathrm{~nm}^{2}\right)$. The force interval width or bin size in the histograms is $10 \mathrm{pN}$.

molecule of $8 \mathrm{~nm}$ and APTES+glutaraldehyde layer thickness of $2 \mathrm{~nm}$, we conclude that essentially an extension of antigen-antibody complex was performed. Different situation was observed for unbinding forces for different antigen-antibody complexes. Two monoclonal antibodies exhibited a narrow distribution of specific unbinding forces. The peak frequency was at $142 \pm 8 \mathrm{pN}$ for the mAb-BSA-1 and at $250 \pm 16 \mathrm{pN}$ for $\mathrm{mAb}-\mathrm{BSA}-33$ (Fig. 3). In contrast, the polyclonal $\mathrm{Ab}$ represents a broad range of forces (from $60 \mathrm{pN}$ until $500 \mathrm{pN}$ ) when tested at "high" (50 or $270 \mu \mathrm{g} / \mathrm{ml}$ ) antibody concentrations (Fig. 4A) but a narrow force distribution, which is variable between different tips, when tested at "low" (10 or $27 \mu \mathrm{g} / \mathrm{ml}$ ) concentration (data not shown). These data were consistent with the heterogeneity of polyclonal Ab compared to monoclonal antibodies. In order to mimic polyclonal 
antibodies, mixtures of two different mAbs were prepared. The data showed that mixing two $\mathrm{mAbs}$ resulted in a force distribution approaching that of the polyclonal Ab (Fig. 4B). The histograms which are represented in this paper were done with covalent functionalization method. We underline that similar FS results (not only the value of unbinding force for one given antigen-antibody pair but also characteristic tip displacement until the moment of the bond rupture and form of an extension curve) were obtained with passive adsorption and covalent attachment methods.

Based on our results we conclude that force spectroscopy can be a valuable tool to measure and recognize the specific antigen-antibody interactions via the specificity and binding efficiency of antibodies.

\section{Acknowledgment}

This work was supported by the Swiss National Science Foundation (grants 2000-61751.00 and 3100-052587.97/1), the Fonds UNIL-EPFL 2000, and the University of Lausanne.

\section{References}

[1] P. Hinterdorfer, W. Baumgartner, H.J. Gruber, K. Schilcher, H. Schindler, Proc. Natl. Acad. Sci. USA 93, 3477 (1996).

[2] J. Dammer, M. Hegner, D. Anselmetti, P. Wagner, D. Dreier, W. Huber, H.-J. Güntherodt, Biophys. J. 70, 2437 (1996).

[3] J.P. Cleveland, S. Manne, D. Bocek, P.K. Hansma, Rev. Sci. Instrum. 64, 403 (1993).

[4] J.L. Hutter, J. Bechhoefer, Rev. Sci. Instrum. 64, 1868 (1993).

[5] U. Jonsson, G. Olofsson, M. Malmqvist, I. Ronnberg, Thin Solid Films 124, 117 (1985).

[6] E.T. Vandenberg, L. Bertilsson, B. Lieberg, K. Uvdal, R. Erlandsson, H. Elwing, I. Lundstrom, J. Colloid Interface Sci. 147, 322 (1991).

[7] Y.L. Lyubchenko, A.A. Gall, L.S. Shlyakhtenko, R.E. Harrington, B.L. Jacobs, P.I. Oden, S.M. Lindsay, J. Biomol. Struct. Dyn. 10, 589 (1992).

[8] Y.L. Lyubchenko, R.E. Blankenship, S.M. Lindsay, L.S. Shlyakhtenko, Scanning Microscopy 10, 97 (1996).

[9] L.S. Shlyakhtenko, A.A. Gall, J.J. Weimer, D.D. Hawn, Y.L. Lyubchenko, Biophys. J. 77, 568 (1999).

[10] E.T. Vandenberg, L. Bertilsson, B. Liedberg, K. Uvdal, R. Erlandsson, H. Elwing, I. Lundström, J. Colloid Interface Sci. 147, 103 (1991).

[11] T. Vallant, H. Brunner, U. Mayer, P. Hoffmann, T. Leitner, R. Resch, G. Friedbacher, J. Phys. Chem. B 102, 7190 (1998).

[12] S. Kasas, B.M. Riederer, S. Catsicas, B. Cappella, G. Dietler, Rev. Sci. Instrum. $71,2082(2000)$. 